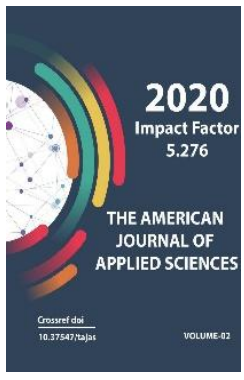

Copyright: Original content from this work may be used under the terms of the creative commons attributes 4.0 licence.

\section{Testing And Determining The Temperature Characteristics Of Heat Pipes (HP)}

\author{
A.A. Kholikov \\ Bukhara Engineering Technological Institute, Bukhara, Uzbekistan \\ F.S.Quldosheva \\ Bukhara Engineering Technological Institute, Bukhara, Uzbekistan
}

\title{
ABSTRACT
}

The article describes the tests and determination of the temperature characteristics of highly efficient heat transfer units (heat pipes (HP)), which can be used in heat and mass transfer devices, for example, in the process of drying vegetables and fruits. To test and determine the temperature characteristics of HP, a test setup for medium-temperature HP was created and a number of tests were carried out to determine how the dependence of the temperature change of the condenser and the HP evaporator at various angles of inclination, the working fluid, body material, HP wick material was selected, the influence of the number of mesh wick and influence of pressure. All characteristics are presented in graphs and tables.

\section{KEYWORDS}

Heat pipe, wick, porosity, mesh number, steel, copper, condenser, evaporator, alcohol, water

\section{INTRODUCTION}

Problems of energy saving processes, as well as the development and implementation of highly efficient equipment and technology for the development of small and medium-sized businesses is an urgent problem.

Energy costs for the implementation of heat and mass transfer processes in the primary processing of raw materials and food products

account for up to $70 \%$ of the total energy expended. Insufficiently efficient use of solar energy and the discharge of the heat exchanger in heat and mass transfer processes, and in production there is no use of highly efficient heat transfer devices - heat 
pipes (HP) [3;-C.13-20, 8;-Ps.7-13, 9;-Ps.24-39, 4;-C.43-50, 6;-C.102-108].

A heat pipe is a heat transfer device capable of transmiHPing large heat powers at low temperature gradients. $\mathrm{HP}$ is a sealed structure made of heat-conducting metal, partially filled with a liquid heat carrier (volatile liquid). Previously, HP was used in space installations for cooling the board, for cooling air in some systems, in computer technology, etc. [1;-C.10-16, 2;-C.15-44,14;,15;,]. The works $[5 ;, 1 ; 2 ; 14 ; 15 ; 10 ; 11 ; 12 ; 13 ;]$ describe in detail the types and work capacity of HP.

\section{THE PURPOSE OF THE STUDY}

In this regard, one of the main tasks is the development of the use of solar energy and waste heat in heat and mass transfer processes of the use of highly efficient heat transfer devices in various production techniques. For this, it is necessary to study the use of highly efficient heat transfer devices in solar heat and mass transfer (in dryers, in evaporators, etc.) installations with a scientifically grounded approach to the design and use of working agents in HP [3; C.76-120, 2 ; -C.182-196,9; -Ps. 40-100,7; -C.6367.].

\section{METHODS AND MATERIALS}

Experimental determination of the characteristics of HP is also a necessary stage

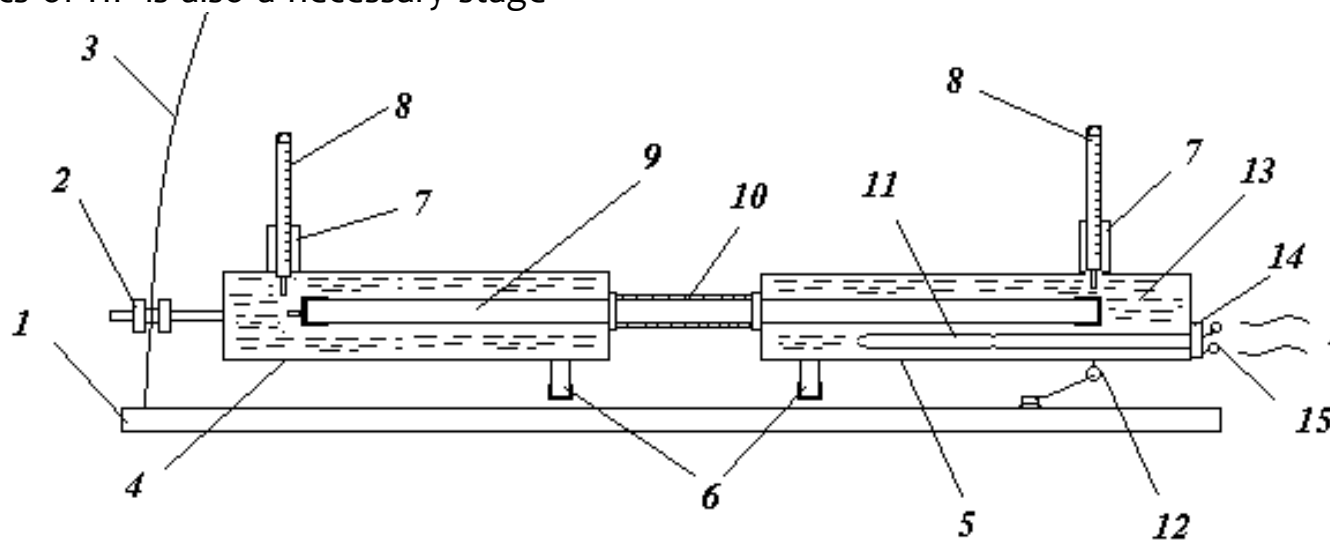

in the work to create a product with the required properties, while it must be said that the interpretation of the test results can be difficult. Test methods for HP, intended for use on heat and mass exchangers, have their own specifics, which is associated with the need to determine the characteristics of HP in zero gravity conditions on the basis of test data performed in technical conditions. Changing the characteristics of HP is relatively simple and requires for its implementation the usual equipment available in any laboratory that studies heat transfer. [1; -C.145-161, 2; C.165-182, 5; -Ps.63-67.].

Measurements are necessary in order to show that the HP meets the design specifications. Limitations of the transmiHPing capacity of the pipe, which form a kind of envelope of the working range of parameters, can also be investigated as well as the degree of isothermality of the pipe. A significant number of factors can be investigated in laboratory tests, including gravity orientation, operating temperature, evaporator heat flow, etc.

To study the influence of the temperature characteristics of $\mathrm{HP}$, we have developed a laboratory experimental setup, which is presented below.

The laboratory experimental setup for HP consists of five main parts, as shown in Fig. 1. 


\section{Fig. 1. Test setup diagram for medium-temperature HP.}

1- bed; 2- mount; 3- tripod; 4,5 - buildings; 6- outflow pipes; 7- drain pipes; 8- thermometers; 9- heat pipe; 10- thermal insulation; 11- heating element; 12- hinge; 13- water; 14- thermostatic device; 15- switch

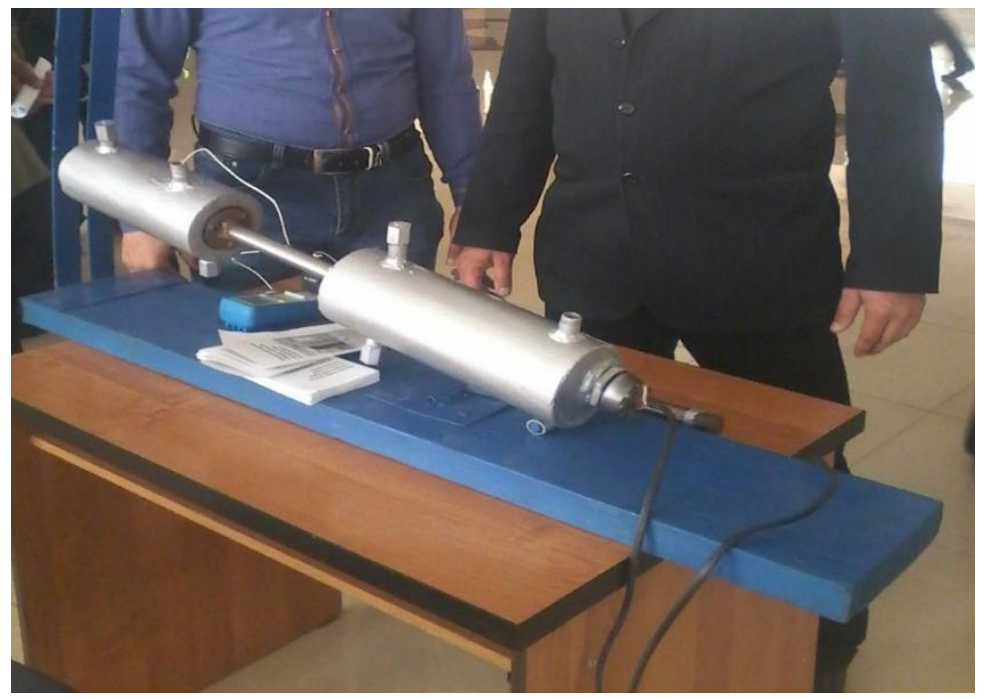

Fig. 2. Test setup for medium temperature HP.

The test setup for medium temperature HP operates as follows. The installation is aHPached to the frame 1 by means of a mount 2 on a tripod 3 and a hinge 12. HP 9 evaporating part is aHPached to the heating body 5 , which consists of a drain pipe 7, a heating element 11, a thermostatic device 14, a switch 15, a thermometer 8 to determine the temperature of the water inside housing 5 , which simulates the flow of thermal energy using the heating element 11. Heating element 11 with the help of switch 15 takes electricity by turning it into heat, which is regulated by a thermostatic device 14, heating the water filled to the body 5 . At the same time, 2,83 liters of water are placed in the body 5, the water heats up to $80-90$ oC while simulating the heat flow energy. HP 9, the evaporating part heats up and takes heat energy, transports it from the inside with the help of the working fluid and, with the help of the CPM (wick), sends heat energy through the steam channel to the part of the HP 9 condenser. Inside the HP 9, the working fluid, having gained thermal energy, converted into steam and through the steam channel is sent to the part of the condenser HP 9 and there, having given off the thermal energy, it condenses into a liquid, then the condensing liquid through a wick and with the help of gravitational forces returns to the evaporative part of HP 9. condensation of the vapor of the working liquid The condensed part of the HP 9 is fixed inside the housing 4 which is filled up 
to 2,33 liters with water to determine how much heat energy is transferred through the water temperature. To check the operation of HP 9 in any orientation of the tilt angle, using the mount 2 and tripod 3, you can change the tilt angle. The graphs of the dependence of the change in the temperature of the condenser and evaporator at various angles of inclination, various materials of wicks and various materials of the HP body are given (Fig. 3, Fig. 4, Fig. 5, Fig. 6, Fig. 7, Fig. 8 ., fig. 9., fig. 10., fig. 11.).

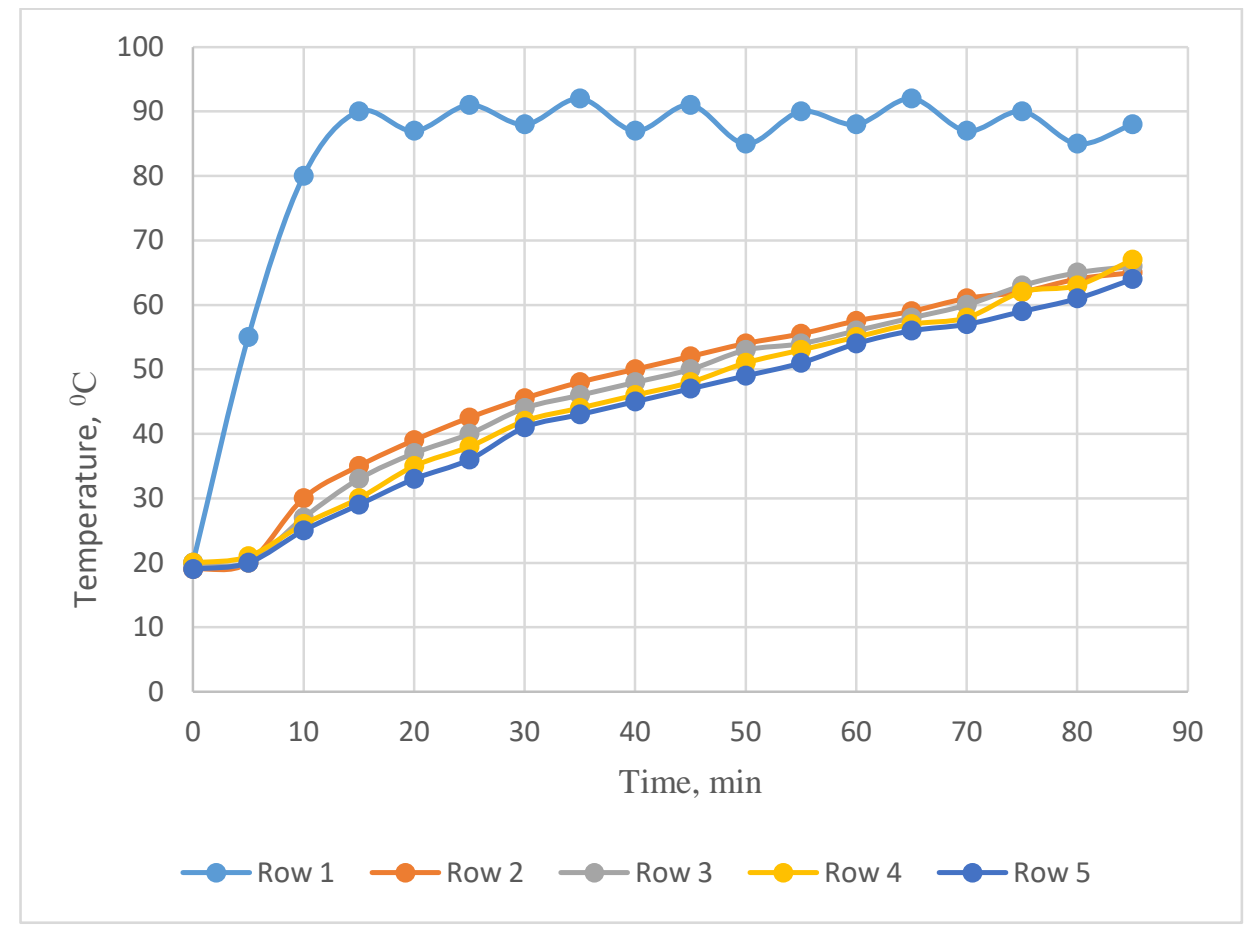

Fig. 3. Dependence of the change in the temperature of the condenser and evaporator at different slope angles of the HP, steel pipe, steel wick, wick thickness $3 \mathrm{~mm}$, mesh number $\mathrm{N}=4800$, working fluid water, HP diameter $\mathrm{d}=\mathbf{2 0} \mathrm{mm}$

Row1 - evaporator temperature, Row2 - tilt angle HP $0^{\circ}$, Row3 - tilt angle HP $30^{\circ}$, Row4 - tilt angle HP $45^{\circ}$, Row 5 - tilt angle HP $60^{\circ}$ 


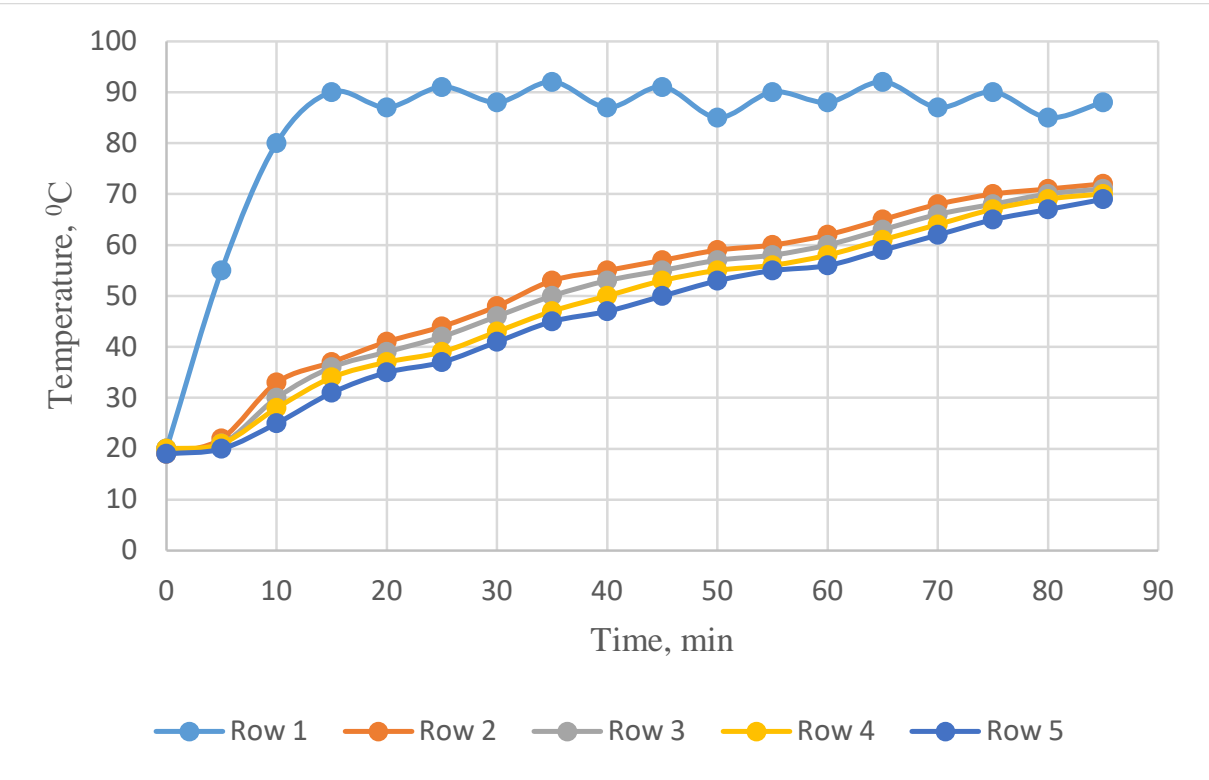

Fig. 4. Dependence of the change in the temperature of the condenser and evaporator at different tilt angles of the HP, steel pipe, steel wick, wick thickness $3 \mathrm{~mm}$, mesh number $\mathrm{N}=7870$, working fluid water, HP diameter $\mathrm{d}=20 \mathrm{~mm}$

Row1 - evaporator temperature, Row2 - tilt angle HP $0^{\circ}$, Row3 - tilt angle HP $30^{\circ}$, Row4 - tilt angle HP $45^{\circ}$, Row 5 - tilt angle HP $60^{\circ}$

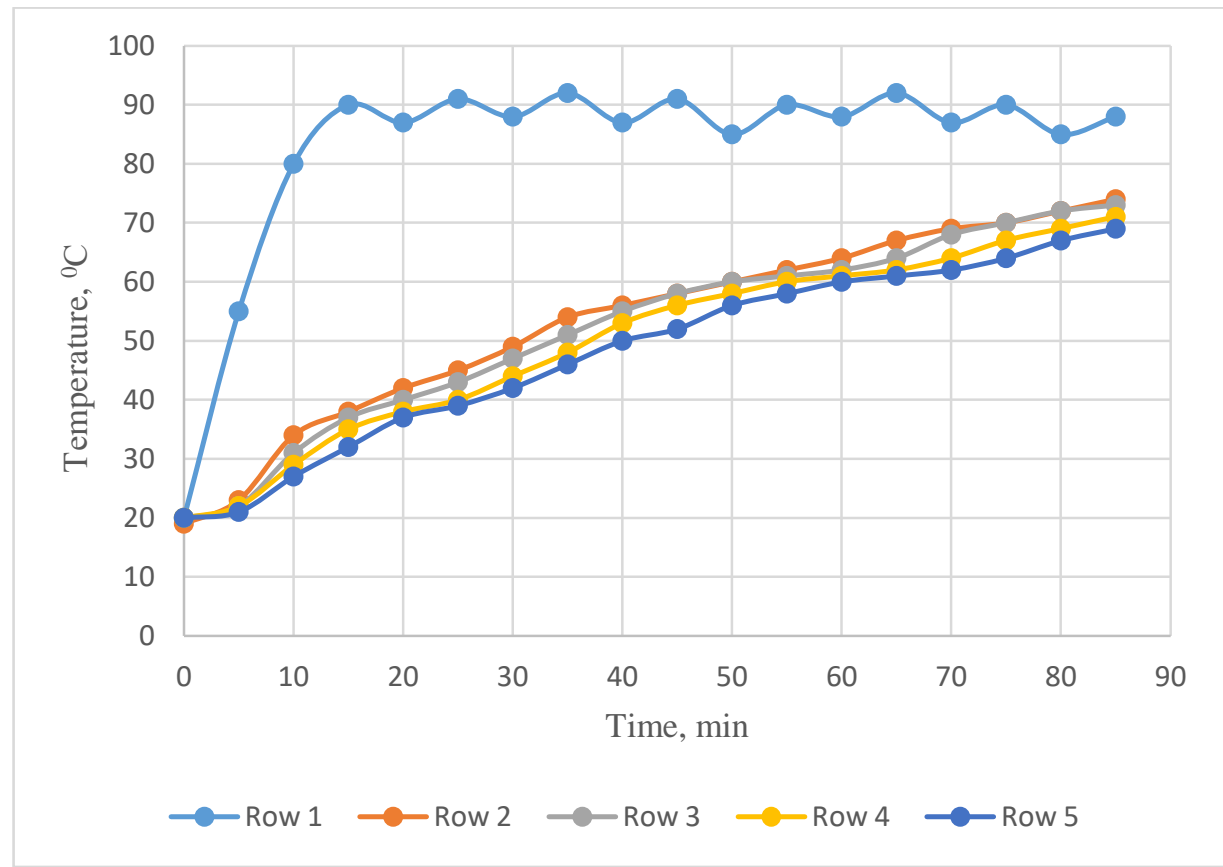

Fig. 5. Dependence of the change in the temperature of the condenser and evaporator at various slope angles of the HP, steel pipe, copper wick, wick thickness $3 \mathrm{~mm}$, mesh number $\mathrm{N}=4800$, working fluid water, HP diameter $\mathrm{d}=20 \mathrm{~mm}$ 
Row1 - evaporator temperature, Row2 - tilt angle $\mathrm{HP}^{\circ}$, Row3 - tilt angle HP $30^{\circ}$, Row4 - tilt angle HP $45^{\circ}$, Row 5 - tilt angle $\mathrm{HP} 60^{\circ}$

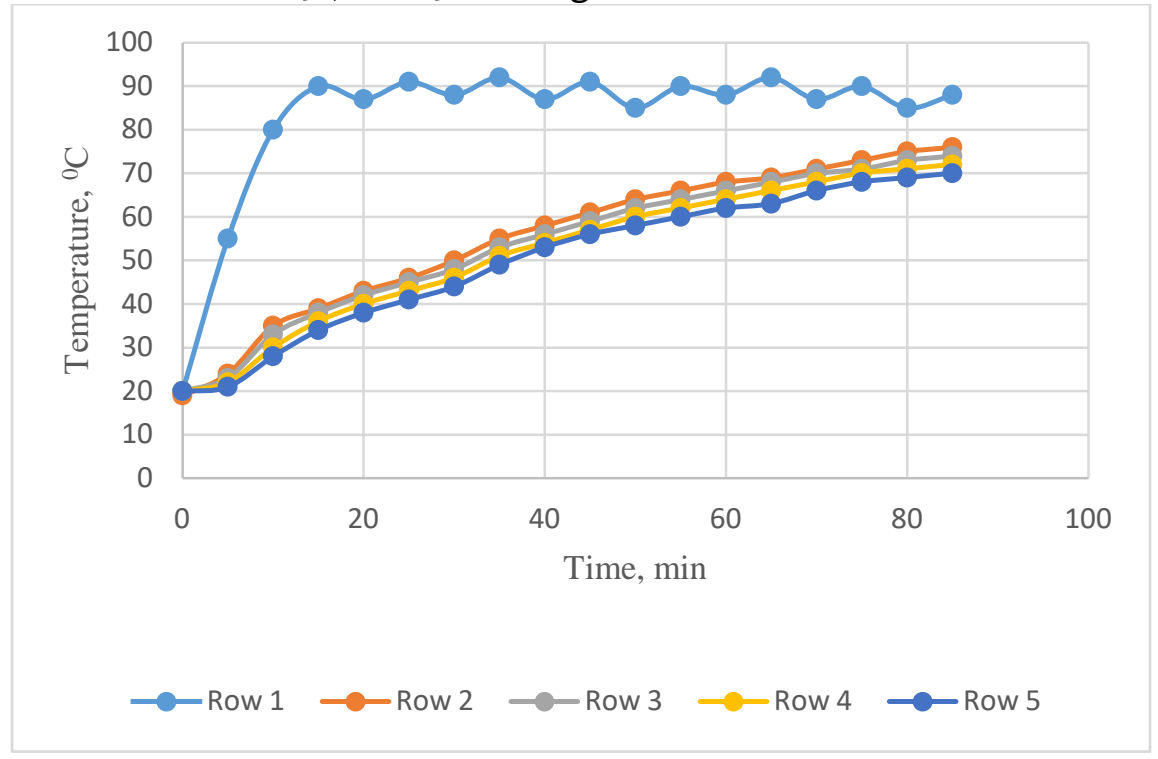

Fig. 6. Dependence of the change in the temperature of the condenser and evaporator at different tilt angles of the HP, steel pipe, copper wick, wick thickness $3 \mathrm{~mm}$, mesh number $\mathrm{N}=7870$, working fluid water, HP diameter $\mathrm{d}=20 \mathrm{~mm}$

Row1 - evaporator temperature, Row2 - tilt angle HP $0^{\circ}$, Row3 - tilt angle HP $30^{\circ}$, Row4 - tilt angle HP $45^{\circ}$, Row 5 - tilt angle $\mathrm{HP} 60^{\circ}$

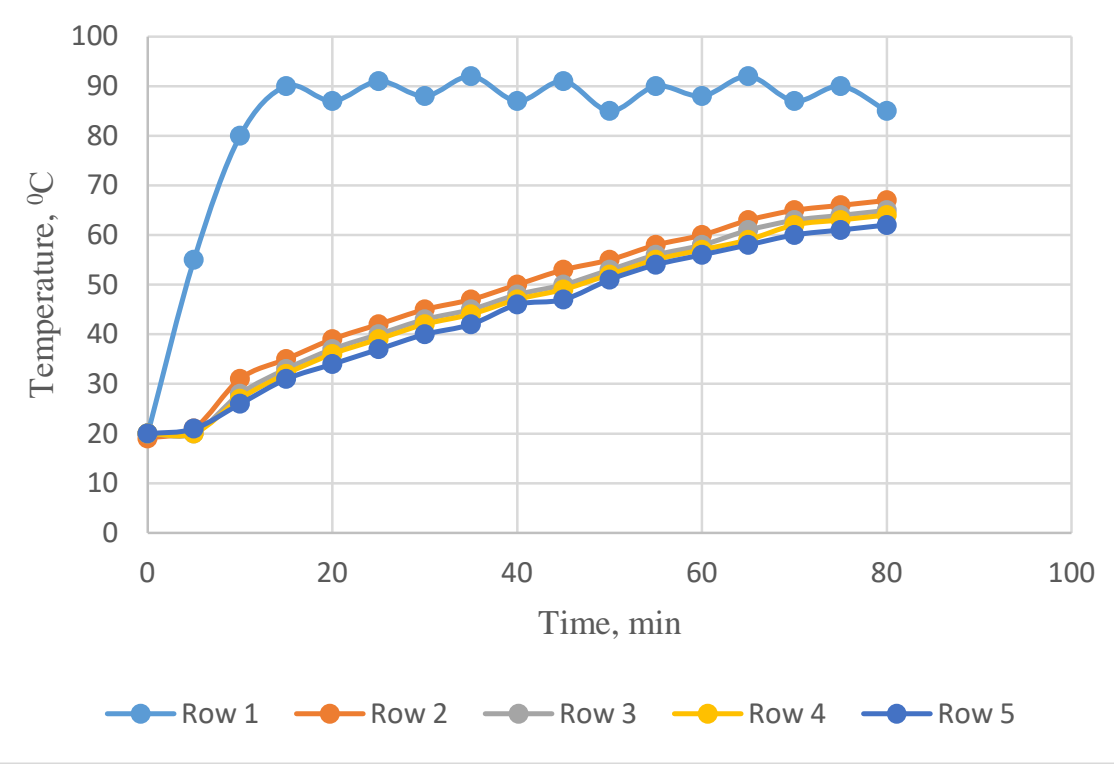

Fig. 7. Dependence of the change in the temperature of the condenser and evaporator at different slope angles of the HP, steel pipe, steel wick, wick thickness $3 \mathrm{~mm}, \mathrm{~N}=4800$, working fluid $70 \%$ alcohol, HP diameter $\mathrm{d}=\mathbf{2 0} \mathrm{mm}$ 
Row1 - evaporator temperature, Row2 - tilt angle $\mathrm{HP} 0^{\circ}$, Row3 - tilt angle HP $30^{\circ}$, Row4 - tilt angle HP $45^{\circ}$, Row 5 - tilt angle $\mathrm{HP} 60^{\circ}$

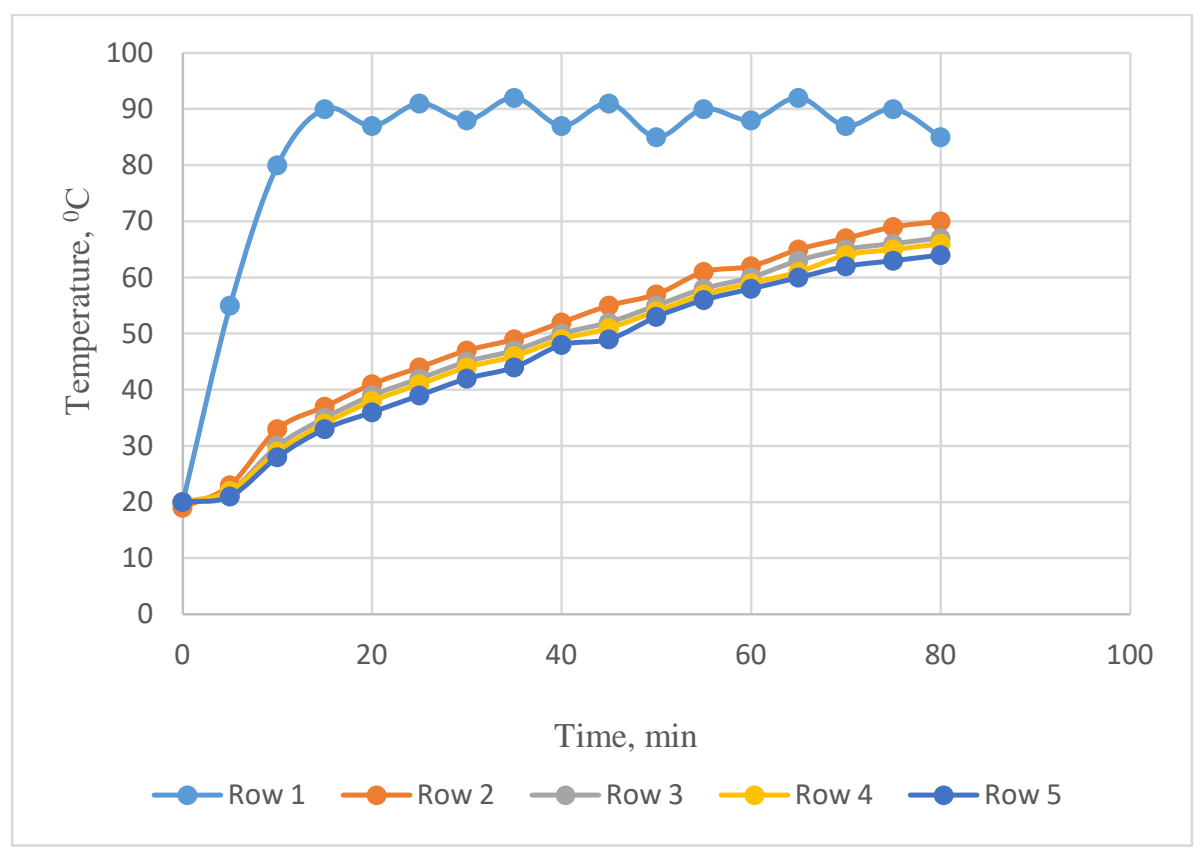

Fig. 8. Dependence of the change in the temperature of the condenser and evaporator at different tilt angles of the HP, steel pipe, steel wick, wick thickness $3 \mathrm{~mm}, \mathrm{~N}=7870$, working fluid $70 \%$ alcohol, HP diameter $\mathrm{d}=\mathbf{2 0} \mathrm{mm}$

Row1 - evaporator temperature, Row2 - tilt angle HP $0^{\circ}$, Row3 - tilt angle HP $30^{\circ}$, Row4 - tilt angle HP $45^{\circ}$, Row 5 - tilt angle HP $60^{\circ}$

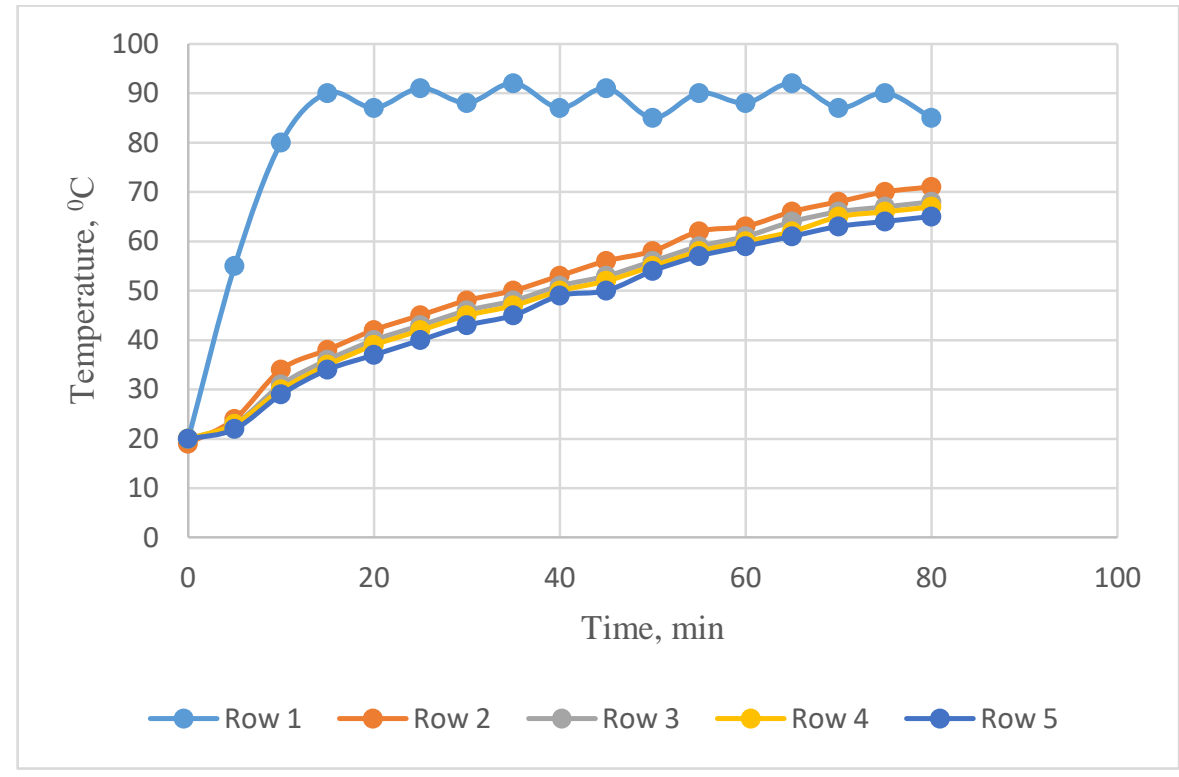


Fig. 9. Dependence of the change in the temperature of the condenser and evaporator at different tilt angles of the HP, steel pipe, copper wick, wick thickness $3 \mathrm{~mm}, \mathrm{~N}=4800$, working fluid $70 \%$ alcohol, HP diameter $\mathrm{d}=\mathbf{2 0} \mathrm{mm}$

Row1 - evaporator temperature, Row2 - tilt angle $\mathrm{HP}^{\circ}$, Row3 - tilt angle HP $30^{\circ}$, Row4 - tilt angle HP $45^{\circ}$, Row 5 - tilt angle HP $60^{\circ}$

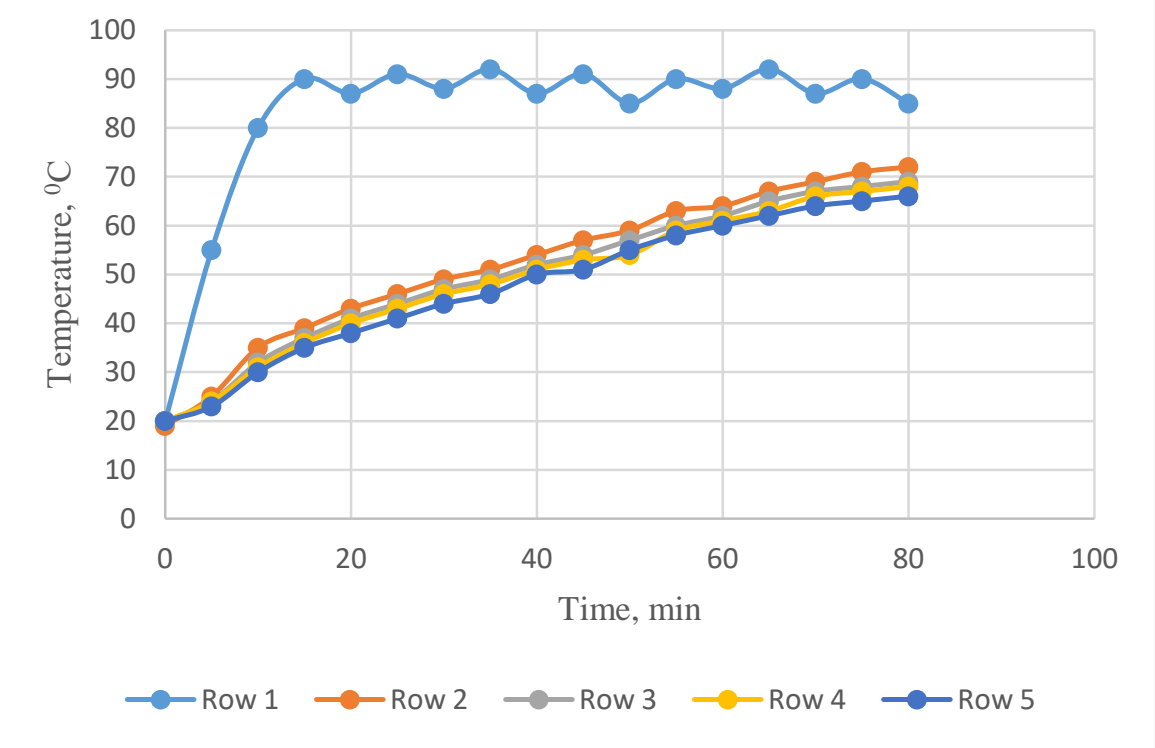

Fig. 10. Dependence of the change in the temperature of the condenser and evaporator at different tilt angles of the HP, steel pipe, copper wick, wick thickness $3 \mathrm{~mm}, \mathrm{~N}=7870$, working fluid $70 \%$ alcohol, HP diameter $\mathrm{d}=\mathbf{2 0} \mathrm{mm}$

Row1 - evaporator temperature, Row2 - tilt angle $\mathrm{HP}^{\circ}$, Row3 - tilt angle HP $30^{\circ}$, Row4 - tilt angle HP $45^{\circ}$, Row 5 - tilt angle HP $60^{\circ}$

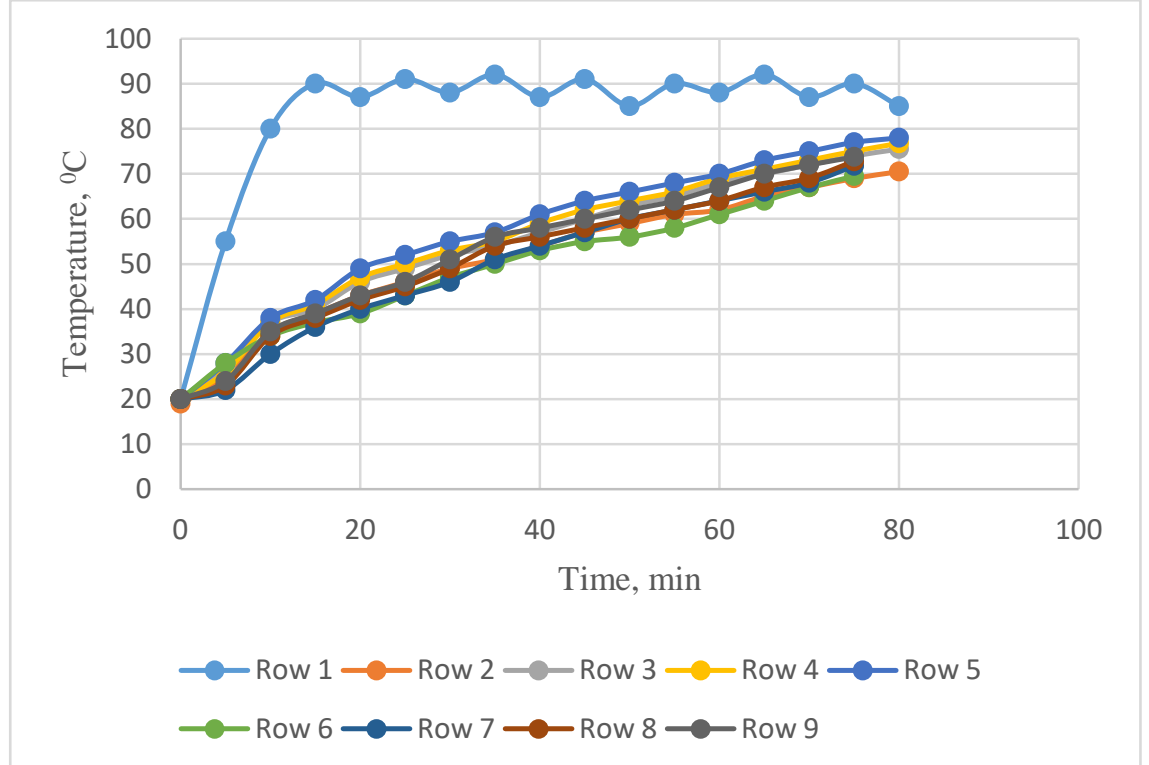

Fig. 11. Dependence of the change in the average temperature of the condenser and evaporator at different slope angles of the HP, copper pipe, wick thickness $3 \mathrm{~mm}$, HP diameter $d=20 \mathrm{~mm}$ 
Row 1 - evaporator water temperature, Row 2 - steel wick, mesh number 4800 , heat carrier water at angles $0^{\circ}, 30^{\circ}, 45^{\circ}, 60^{\circ}$, Row 3 - steel wick, mesh number 7870 , heat carrier water at angles $0^{\circ}, 30^{\circ}$, $45^{\circ}, 60^{\circ}$, Row 4- copper wick, mesh number 4800 , heat carrier water at angles $0^{\circ}, 30^{\circ}, 45^{\circ}, 60^{\circ}$, Row 5 - copper wick, mesh number 7870 , heat carrier water at angles $0^{\circ}, 30^{\circ}, 45^{\circ}, 60^{\circ}$, Row 6 - steel wick, mesh number 4800 , heat carrier alcohol $70 \%$ at angles $0^{\circ}, 30^{\circ}, 45^{\circ}, 60^{\circ}$, Row 7 - steel wick, mesh number 7870 , heat carrier alcohol $70 \%$ at angles $0^{\circ}, 30^{\circ}, 45^{\circ}, 60^{\circ}$, Row 8 - copper wick, mesh number 4800 , heat carrier alcohol $70 \%$ at corners $0^{\circ}, 30^{\circ}, 45^{\circ}, 60^{\circ}$ Row 9 - copper wick, mesh number 7870 , heat carrier $70 \%$ alcohol at angles $0^{\circ}, 30^{\circ}, 45^{\circ}, 60^{\circ}$

\section{RESULTS AND DISCUSSION}

It can be seen from the graphs that the slope angle of the HP has liHPle effect on the increase in the water temperature of the case 5 from it, it can be concluded that the main influence on the flow of thermal energy will be affected by the working fluid (coolant), the thickness of the wick, the thermal conductivity of the coolant, the heat transfer coefficient of the HP material and the wick. The above graphs are only for steel HP, all experiments of HP are charged with coolants at atmospheric pressure for comparison with copper HPs are shown in the following table. All experiments were carried out with a HP length of $730-770 \mathrm{~mm}$.

Table 1.

\begin{tabular}{|c|c|c|c|c|c|}
\hline № & $\begin{array}{c}\text { HP material, with a wick } \\
\text { thickness of 3mm }\end{array}$ & $\begin{array}{c}\text { Mesh } \\
\text { number, N }\end{array}$ & $\begin{array}{c}\text { Heat } \\
\text { carrier }\end{array}$ & $\begin{array}{c}\text { Time for } \\
\text { increasing the } \\
\text { water } \\
\text { temperature of } \\
\text { the condenser } \\
\text { body, min }\end{array}$ & $\begin{array}{c}\text { Average water } \\
\text { temperature of } \\
\text { the condenser } \\
\text { body at slope } \\
\text { angles in time, } \\
{ }^{\circ} \mathrm{C}\end{array}$ \\
\hline 1. & Steel pipe, steel wick & 4800 & water & 85 & 65,5 \\
\hline 2 & Steel pipe, steel wick & 7870 & water & 85 & 70,5 \\
\hline 3 & Steel pipe, copper wick & 4800 & water & 85 & 71,75 \\
\hline 4 & Steel pipe, copper wick & 7870 & water & 85 & 73 \\
\hline 5 & Steel pipe, steel wick & 4800 & alcohol, & 80 & 64,5 \\
\hline 6 & Steel pipe, steel wick & 7870 & alcohol, & 80 & 67,75 \\
\hline 7 & Steel pipe, copper wick & 4800 & alcohol, & 80 & \\
\hline & & & $70 \%$ & & 6 \\
\hline
\end{tabular}


The American Journal of Applied Sciences

(ISSN - 2689-0992)

Published: November 30, 2020 | Pages: 151-166

Doi: https://doi.org/10.37547/tajas/Volume02Issue11-27

\begin{tabular}{|c|c|c|c|c|c|}
\hline 8 & Steel pipe, copper wick & 7870 & alcohol, & 80 & 68,75 \\
\hline 9 & Copper pipe, steel wick & 4800 & water & 80 & 70,5 \\
\hline 10 & Copper pipe, steel wick & 7870 & water & 80 & 75,5 \\
\hline 11 & Copper pipe, copper wick & 4800 & water & 80 & 76,75 \\
\hline 12 & Copper pipe, copper wick & 7870 & water & 80 & 78 \\
\hline 13 & Copper pipe, steel wick & 4800 & alcohol, & 75 & 69,5 \\
\hline 14 & Copper pipe, steel wick & 7870 & alcohol, & 75 & 71,75 \\
& & & $70 \%$ & & 72,75 \\
\hline 15 & Copper pipe, copper wick & 4800 & alcohol, & 75 & 73,75 \\
\hline 16 & Copper pipe, copper wick & 7870 & alcohol, & 75 & \\
\hline
\end{tabular}

Based on these data, it is necessary to choose for our purposes which is possible to use a solar dryer using HP. We take pipes made of copper and copper meshes for analysis. The thermal conductivity of copper pipes and grids is very good, but it is not easy to find the required diameter with the pipe thickness and the wire diameter of the wick grids, it is oxidized with water, the mechanical processing of copper pipes is difficult. Now the coolant water is the cheapest coolant, the heat capacity is good, but it oxidizes with metal contact, the boiling point is 100 degrees for our purposes, this coolant is not suitable. What remains is a steel pipe and a steel mesh for wicks, the thermal conductivity of this material is slightly less than copper is widely accepted in production and it is not difficult to find such materials and is very easy to machine. Alcohol 70\%, the heat capacity of this coolant is less than water, but it is easy to find, less oxidized with metals than water. From the table, we can conclude that for our case we need to choose a HP steel pipe, a steel wick with a mesh number of 7870 and a coolant $70 \%$ alcohol.

The selected HP as a heat carrier of $70 \%$ alcohol operates on a temperature gradient above $80 \mathrm{OC}$ at atmospheric pressure inside the HP. The boiling point of $70 \%$ of that alcohol is $80.72 \mathrm{OC}$, in order to reduce the boiling point, you need to create a vacuum inside the HP. 


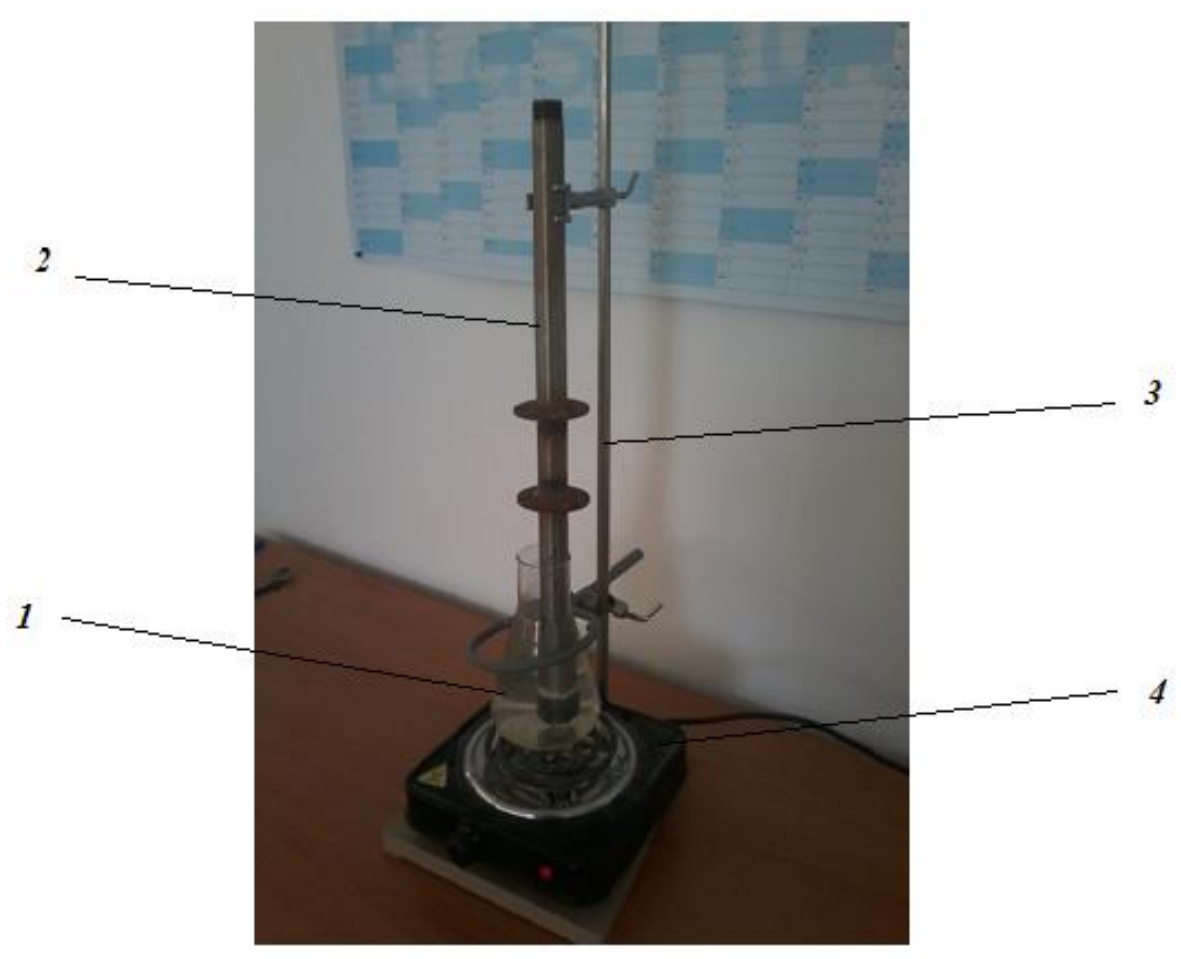

Fig. 12. Laboratory setup for vacuum inside HP

1-heat-resistant flask, 2-HP, 3-tripod, 4- heater.

In order to create a vacuum inside the HP, we need a heat-resistant flask 1 , a stand 3 , and a heating element 4 is shown in Fig. First, water is poured into flask 1 with the help of a heating element, we boil water. At the same time, HP 2 with an open top cover is fixed to the tripod 3 , and the coolant is poured inside the HP 2 and the coolant is brought to a boil until the coolant vapor escaped from the open cover. In the literatures $[1 ;, 2 ;$.$] , the coolant is poured$ $25-30 \%$ of the total volume and we will pour in the same way. The steam displaces the air and pushes the full weight of the volume with the heat carrier vapor. Leaving the vapor from the top cover of the HP heats the weight of the case of the HP, when the temperature of the case reaches the desired temperature, that is, $60-900 \mathrm{C}$, then the top cover of the HP can be closed hermetically. After the HP is detached from the tripod and you need to wait until it cools down to room temperature. After closing the HP cover, the coolant vapor condenses inside the HP and creates a vacuum. Now we need to check the HP if we have a vacuum inside the HP. To check the HP vacuum, hold the HP vertically by hand and shake it sharply up and down. When the HP is shaking down from the inside, a sound is generated as if a metal ball is loose inside the $\mathrm{HP}$, this means that a vacuum has been created inside the HP, and if this sound does not exist, then the HP has a leak and will not work. Scientific research based on laboratory data, the vacuum created inside the HP reached $\mathrm{P}=0,15 \mathrm{~atm}$. 


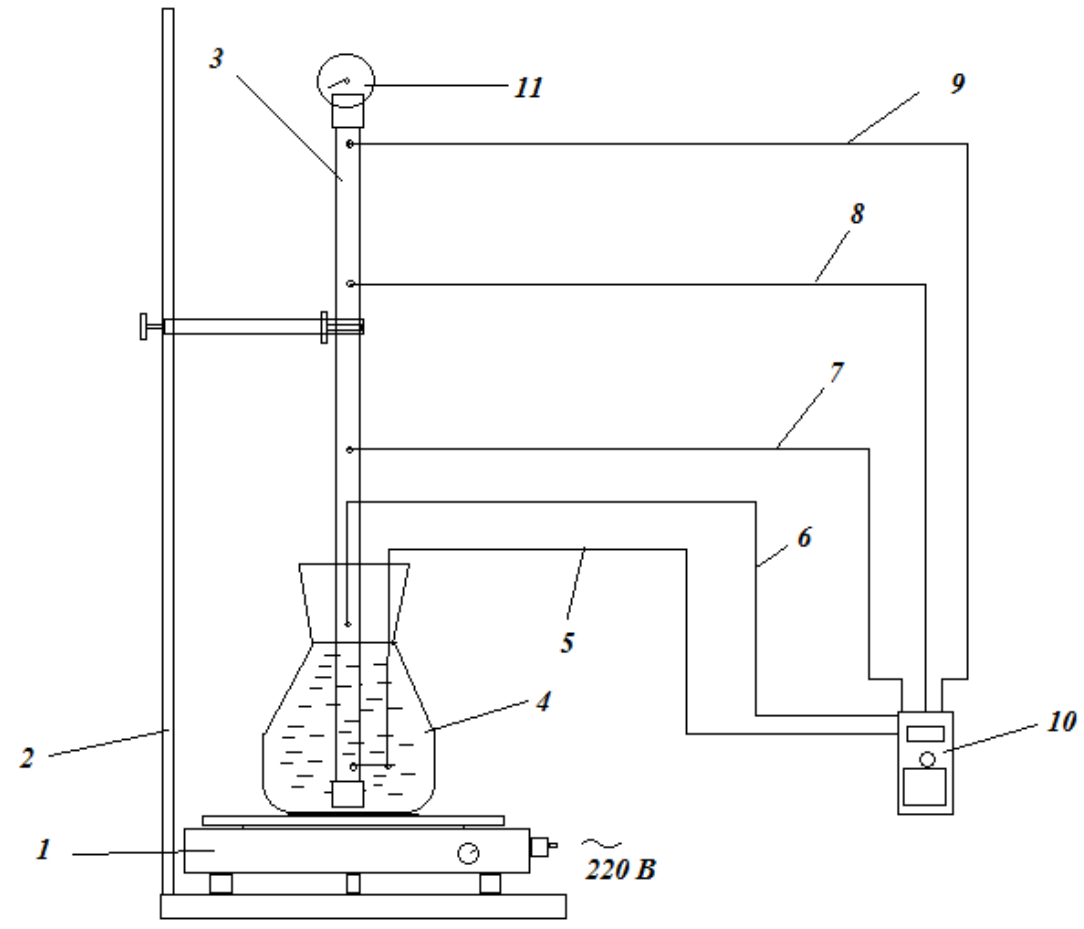

Fig.13. Schematic of an experimental setup for testing the performance of a HP at a pressure of $P=$

\section{$0.15 \mathrm{~atm}$. inside the HP.}

1-heating element, 2-tripod, 3-HP, 4-heat-resistant flask, 5,6,7,8,9- thermocouples, 10- multimeter, 11manometer.

To test the performance of the HP at a pressure of $P=0,15$ atm. inside the HP is shown in Fig. HP 3 is fixed on a tripod 2, the lower part of HP 3 is inside a heat-resistant flask 4 which is filled with water. Water is heated by means of heating element 1 . In the case of HP 35 thermocouples are fixed in different places, divided equally, which are measured using a multimeter in time. Proceeding from this, the heating element 1 heats and boils the water, which simulates the flow of heat energy and gives in the lower part of the HP 3. After that, it is necessary to determine the temperature change over time of the various parts that are fixed thermocouples and the change in pressure inside the HP 3. The graph of experiments is shown in the following figures (fig. 14., fig. 15. fig. 16.). 


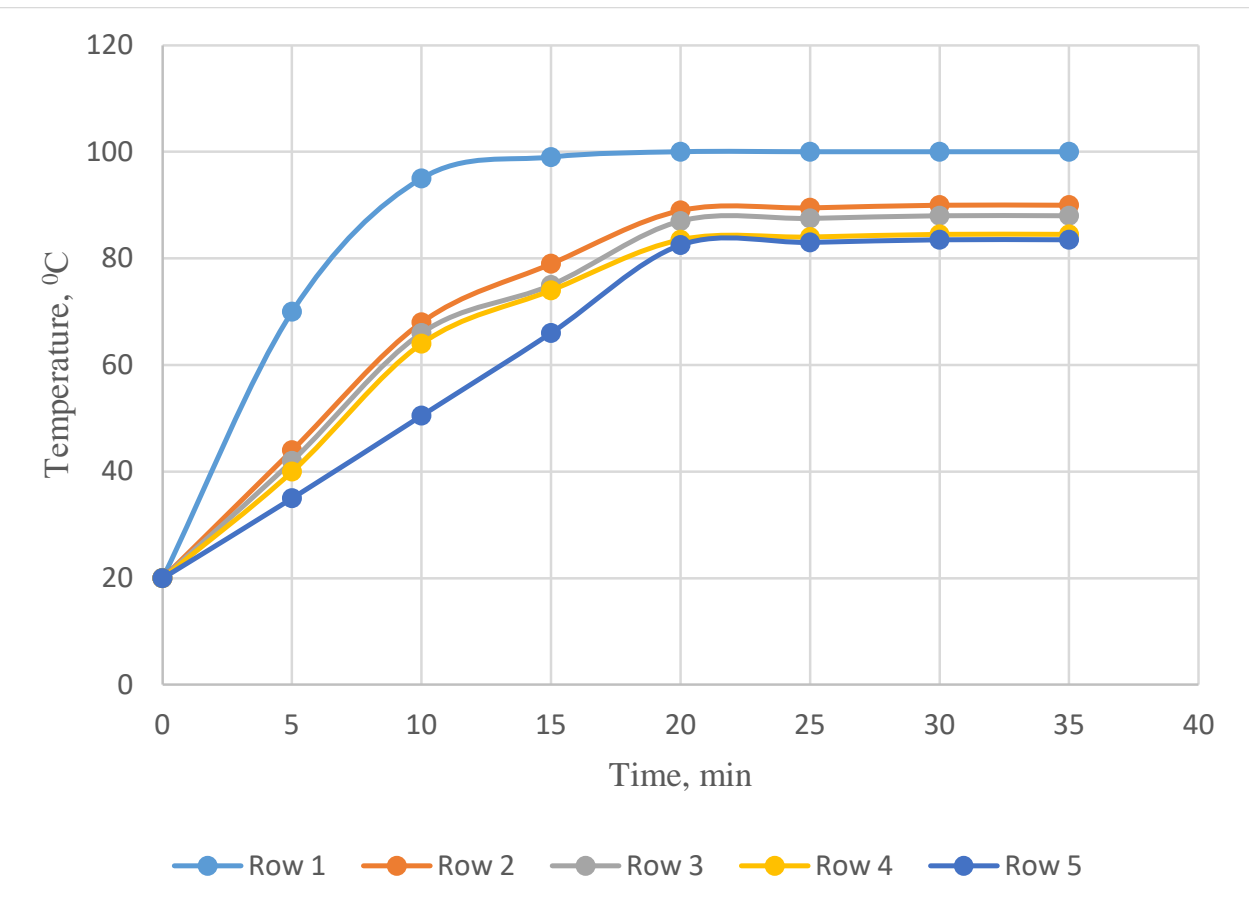

Fig. 14. Dependences of the change in the temperature of the HP at various points at a vacuum $\mathrm{P}=\mathbf{0 . 1 5} \mathrm{atm}$. Steel pipe, steel wick, heat carrier alcohol 70\%, HP diameter $\mathbf{d}=\mathbf{2 0} \mathrm{mm}$ Row1- thermocouple 5, Row2- thermocouple 6, Row3- thermocouple 7, Row4- thermocouple 8, Row5- thermocouple 9,

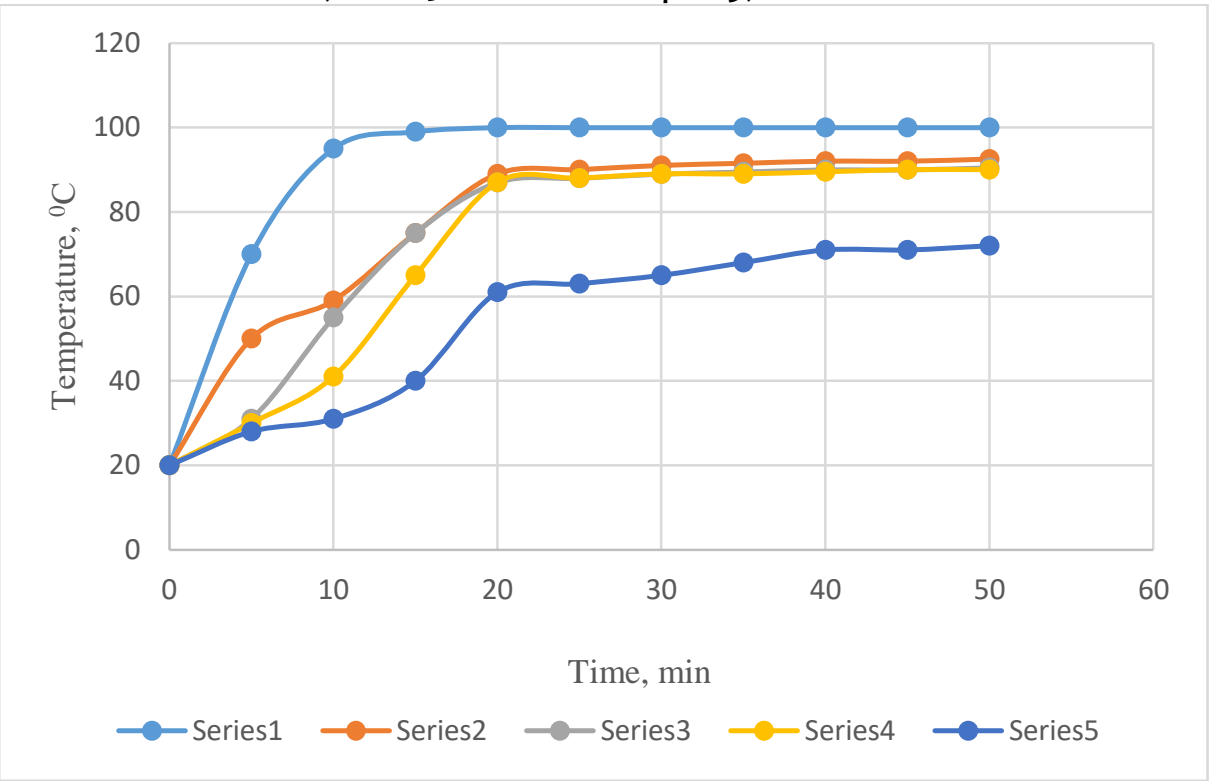

Fig. 15. Dependence of the change in the temperature of the HP at various points at a vacuum $\mathrm{P}=\mathbf{0 . 1 5} \mathrm{atm}$. Steel pipe, steel wick, distilled water heat carrier, HP diameter $\mathbf{d}=\mathbf{2 0}$ $\mathrm{mm}$ 
Row1- thermocouple 5, Row2- thermocouple 6, Row3- thermocouple 7, Row4- thermocouple 8, Row5- thermocouple 9,

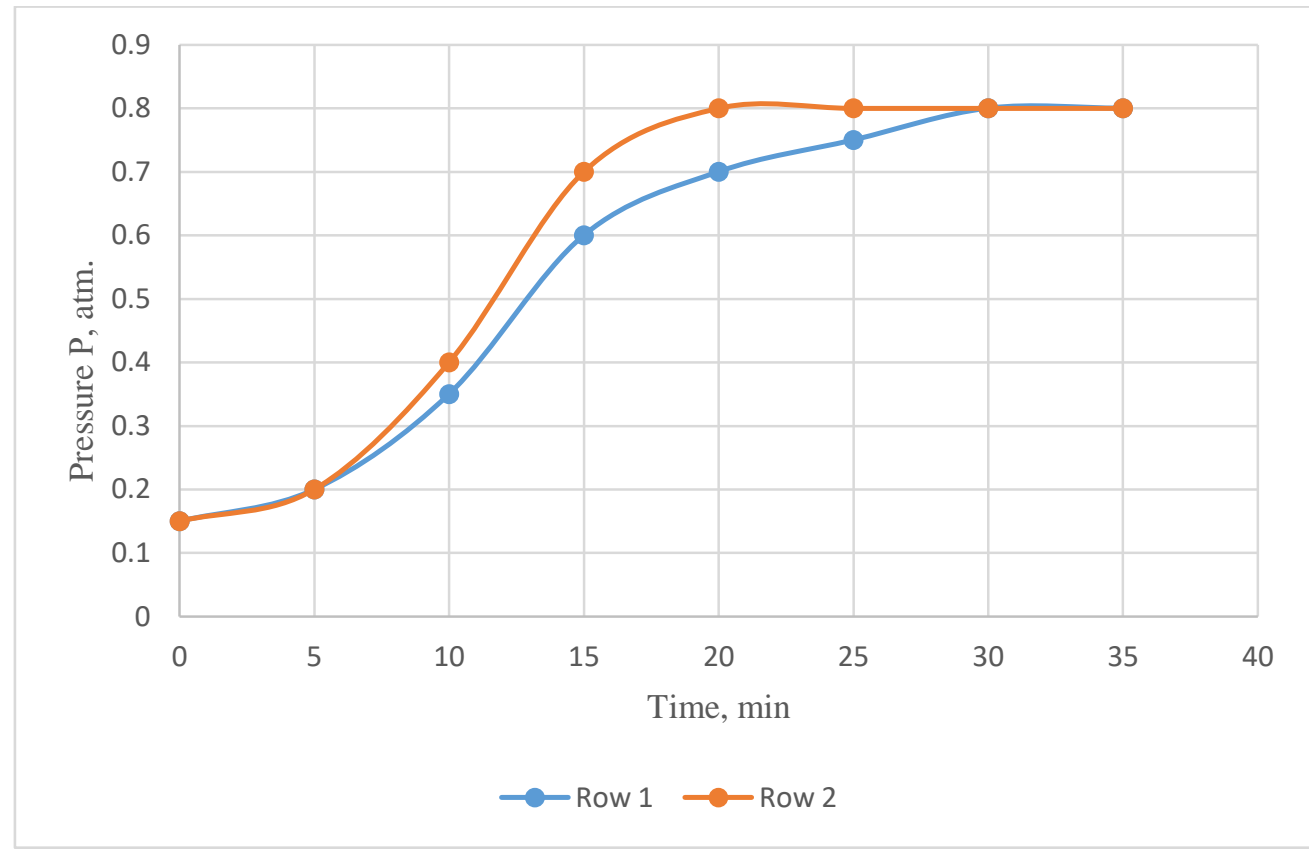

Fig. 16. Dependence of the pressure change inside the HP of the temperature pair. Steel pipe, steel wick, distilled water and 70\% alcohol coolant, HP diameter $\mathbf{d}=\mathbf{2 0} \mathbf{~ m m}$ Row1 - heat carrier 70\% alcohol, Row2 - heat carrier distilled water
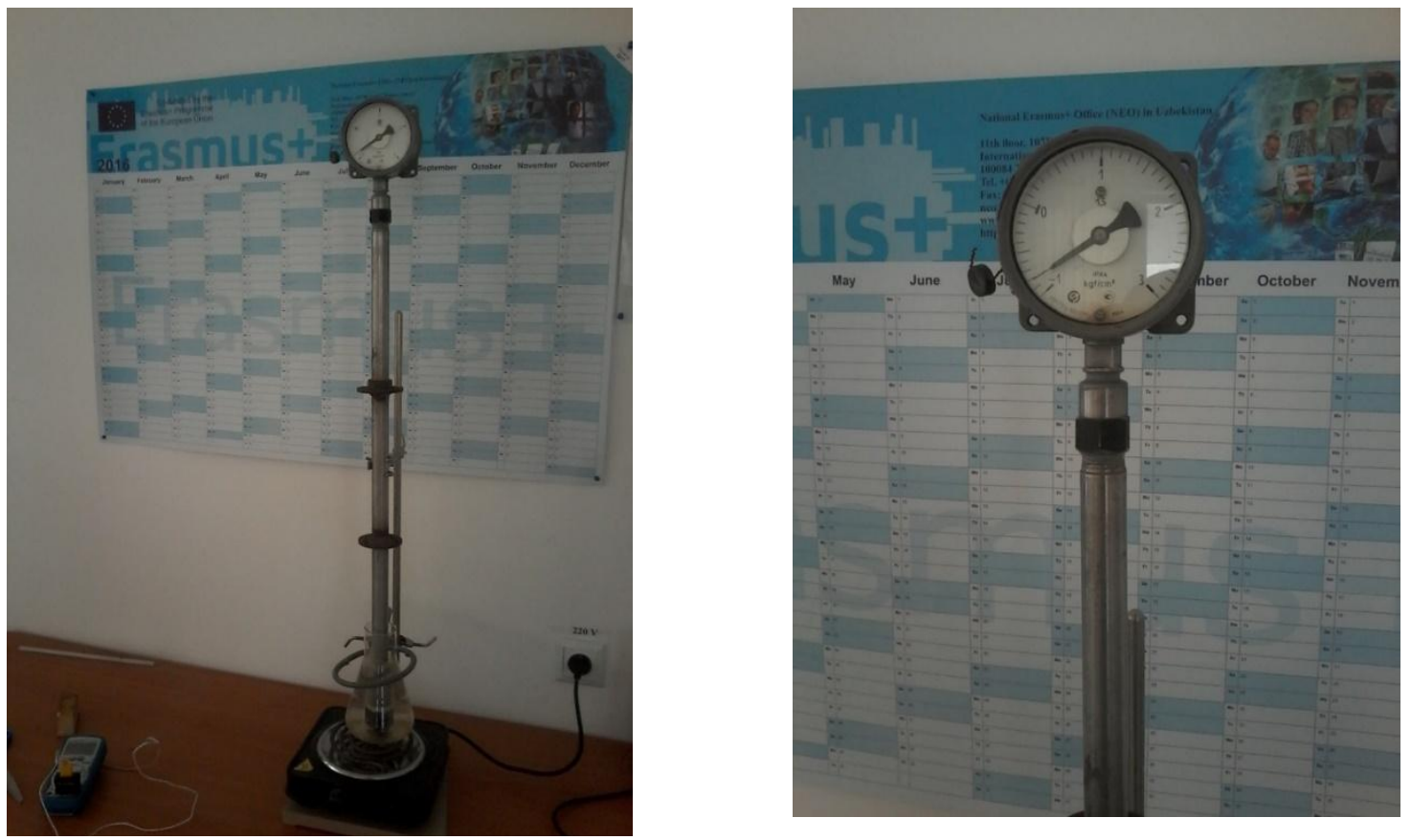


\section{Fig. 17. Experimental setup for checking the performance of HP at a pressure of $P=0.15$ atm. inside the HP.}

Table 2.

\begin{tabular}{|c|c|c|c|}
\hline \multicolumn{2}{|c|}{$\begin{array}{r}\text { Dependence of the boiling point of ethyl } \\
\text { alcohol at different pressures }\end{array}$} & \multicolumn{2}{|c|}{$\begin{array}{r}\text { Dependence of the boiling point of } 70 \% \\
\text { ethyl alcohol at various pressures }\end{array}$} \\
\hline $\begin{array}{c}\text { Pressure, } \\
\mathrm{mmHg}\end{array}$ & Boiling point, ${ }^{\circ} \mathrm{C}$ & $\begin{array}{c}\text { Pressure, } \\
\mathrm{mmHg}\end{array}$ & Boiling point, ${ }^{\circ} \mathrm{C}$ \\
\hline 100 & 34,2 & 100 & 42,4 \\
\hline 150 & 42 & 200 & 49,8 \\
\hline 200 & 47,8 & 400 & 64,5 \\
\hline 400 & 62,8 & 600 & 74,2 \\
\hline 760 & 78,15 & 760 & 80 \\
\hline
\end{tabular}

Tests of the thermal characteristics of the HP were carried out on a test laboratory setup where it is shown in Fig. 2 and Fig. 12. All experiments were carried out with a HP length from $730-770 \mathrm{~mm}$ with a pipe diameter of $20 \mathrm{~mm}$. Experimental data showed that the use of HP for drying fruits and vegetables, the material of the HP body and the material of the wick must be selected from steel and the heat carrier $70 \%$ ethanol and filling this coolant, as shown in the literature, must fill 25$30 \%$ of the total capacity inside the HP. The tilt angle of the HP at different angles has little effect on heat transfer. It was determined that the temperature in the condenser part of the HP is less than $10-15 \%$ of the evaporative part of the HP. This can explain the influence of the environment, thermal resistance of metals, hydraulic resistance of vapor and liquid. The selected HP as a heat carrier of $70 \%$ alcohol operates on a temperature gradient above $80 \mathrm{OC}$ at atmospheric pressure inside the HP. The boiling point of $70 \%$ of that alcohol is $80.72 \mathrm{OC}$, in order to reduce the boiling point, a vacuum is created inside the HP to $0,15 \mathrm{~atm}$. At this pressure, the boiling point inside the HP of $70 \%$ of that ethyl alcohol reached $46.10 \mathrm{C}$ and this temperature is suitable in our case. The change in pressure at a temperature gradient over time in the maximum mode changed from 0,15 atm. up to $0,8 \mathrm{~atm}$.

\section{REFERENCES}

1. Dan.P.D., Ray D.A. Heat pipes. Translated from English by Yu.A. Zeigarnik: $-M$.: Energiya, 1979, $272 \mathrm{p}$.

2. Chi.S. Heat pipes: Theory and practice / Translated from English. V. Ya. Sidorov. -M .: Mechanical engineering, 1981.-207 p.

3. Safarov A.F., Gafurov K.Kh., Khikmatov D.N., Kholikov A.A. Energy saving technologies in the processing of fruits and vegetables. Bukhara. Publisher: Durdona, 2013 .-- 248 p.

4. Ibragimov R.R., Kholikov A.A., Abdurakhmonov O.R., Kobilov Kh.Kh. Ways to solve the energy costs of technological processes. Moscow. I Bulletin of the development of science and education. No. 3 2003, p. 43-50.

5. Alijon Abdiraupovich Kholikov, Hamrokul Kuvandikovich Sayidov, Murodjon Hamidjonovich Beshimov. Analysis of Mathematical Models and Influence of Constructions of High Efficient Heat Transfer Devices. / Mathematical Modeling and Applications.Science Publishing Group Ps: 63-67. Published Online: Dec. 13, 2017.

6. Kholikov A.A. Issiklik қuvurlarda nostationar energy of tashishni tқіқ ethish. 
"Fan va tekhnologlar taragiyoti" (Development of science and technology) Bukhoro 2018y 4 kism.

7. Kholikov A.A., Mirzaeva Sh.U., Rakhmatov U.R. Calculation of a convective drying plant using heat pipes. "UNIVERSUM: TECHNICAL SCIENCES" Electronic scientific journal No. 10 (67), Moscow 2019, pp.6367.

8. Gafurov K., Hikmatov D.,Kholiqov A.A.,Safarov O. Energy resourse-saving technologies in processing of fruits and vegetables. Monograph. Publishing hause "Durdona" Bukhara. Protocol №3 dated 29.11.2019. “Шарқ -Бухоро” МЧЖ босмахоноаси 2019й.

9. Gafurov K.Kh., Hikmatov D.N., Kholikov A.A., Safarov O.F. Application of heat pumps and heat pipes in the food industry. Monograph. Publishing hause "Durdona" Bukhara. Protocol №3 dated 01.07.2020. “Шарқ -Бухоро” МЧЖ босмахоноаси 2020 й.

10. GongmingXinaPengZhangaYanChenaLinC hengabTengHuangbHongyiYinb.

Development of composite wicks having different thermal conductivities for loop heat pipes. Applied Thermal Engineering.Volume 136, 25 May 2018, Pages 229-236

11. MahboobeMahdaviaSaeedTiariaSvenDe SchampheleirebSonggangQiuc.

Experimen-tal study of the thermal characteristics of a heat pipe. Experimental Thermal and Fluid Science.Volume 93, May 2018, Pages 292304

12. PrameshRegmiHarrisWong. Heat and mass transfer in a cylindrical heat pipe with a circular-capillary wick under small imposed temperature differences. International Journal of Heat and Mass Transfer .Volume 120, May 2018, Pages 228-240

13. M.VijayakumaraP.NavaneethakrishnanbG. KumaresancR.Kamatchid. A study on heat transfer characteristics of inclined copper sintered wick heat pipe using surfactant free $\mathrm{CuO}$ and $\mathrm{Al}_{2} \mathrm{O}_{3}$ nanofluids. Journal of the Taiwan Institute of Chemical Engineers.Volume 81, December 2017, Pages 190-198

14. hHPp://www.trizminsk.org/e/21102100.htm

15. hHPp://engineeringsystems.ru/teploviyetrubi 